\title{
SOME STABLE RESULTS ON THE COHOMOLOGY OF THE CLASSICAL INFINITE-DIMENSIONAL LIE ALGEBRAS
}

\author{
BY \\ VICTOR GUILLEMIN AND STEVEN SHNIDER(1)
}

\begin{abstract}
In this paper we compute the cohomology of various classical infinitedimensional Lie algebras generalizing results of Gel'fand-Fuks for the Lie algebra of all formal power series vector fields.
\end{abstract}

1. Let $k$ be a field of characteristic zero and $x_{1}, x_{2}, \ldots, y_{1}, y_{2}, \ldots$, etc. indeterminants. For us the classical infinite-dimensional Lie algebras will mean simply the Lie algebras occurring on the following list.

(I) Formal power series vector fields. This is just all expressions of the form $\sum_{i=1}^{n} f_{i}\left(\partial / \partial x_{i}\right)$, where $f_{i} \in k\left[\left[x_{1}, \ldots, x_{n}\right]\right]$. The bracket is the usual Lie bracket.

(II) $n$ Divergence zero formal power series vector fields. The subalgebra of (I) consisting of all $\vec{f}=\sum f_{i}\left(\partial / \partial x_{i}\right)$ for which $\operatorname{div} \vec{f}=\sum\left(\partial f_{i} / \partial x_{i}\right)=0$.

(III) $)_{n}$ Divergence constant formal power series vector fields. The subalgebra of (I) consisting of all $\vec{f}$ for which $\operatorname{div} \vec{f} \in k$.

$(I V)_{2 n}$ The Poisson algebra. As a vector space this is $k\left[\left[x_{1}, \ldots, x_{n}, y_{1}, \ldots, y_{n}\right]\right]$, and the bracket operation is the Poisson bracket:

$$
\{f, g\}=\sum \frac{\partial f}{\partial x_{i}} \frac{\partial g}{\partial y_{i}}-\frac{\partial g}{\partial x_{i}} \frac{\partial f}{\partial y_{i}} .
$$

$(\mathrm{V})_{2 n}$ The Hamiltonian algebra. The Poisson algebra over its one-dimensional center.

$(\mathrm{VI})_{2 n}$ The derivation algebra of the Hamiltonian algebra. Since there is just one outer derivation, this contains the Hamiltonian algebra as a codimension one ideal.

$(\mathrm{VII})_{2 n+1}$ The contact algebra. As a vector space this is $k\left[\left[x_{1}, \ldots, x_{n}, y_{1}, \ldots\right.\right.$, $y_{n} z$ ]]. The bracket operation is the Lagrange bracket:

$$
[f, g]=\{f, g\}_{x, y}+\frac{\partial f}{\partial z}\left(g-\sum y_{i} \frac{\partial g}{\partial y_{i}}\right)-\frac{\partial g}{\partial z}\left(f-\sum y_{i} \frac{\partial f}{\partial y_{i}}\right) .
$$

In this paper we will compute the cohomology of each of these algebras (with coefficients in $k$ ) for the range $0 \leq i \leq n$. It turns out that in this range the answer in each case is independent of $n$ and rather simple. In certain cases our

Received by the editors May 28, 1972

AMS (MOS) subject classifications (1970). Primary $18 \mathrm{H} 25$.

(') The research for this work was partially supported by NSF grants P22927 and GP-31359X respectively.

Copyright $\odot 1973$, American Mathematical Society 
results can be considerably improved. For example Gel'fand and Fuks computed the entire cohomology ring of $(\mathrm{I})_{n}$ in [2]; and, with slight modifications, their computation also works for (III) $n$. (See [7].) A complete picture of the range $0 \leq i \leq 2 n$ has been obtained by the second author for all the above examples except (II) $)_{n}$ and (VII) $)_{2 n+1}$. (See [7] and the remarks at the end of §4.) However, these improved results seem to require much more delicate arguments than ours. (2)

2. Let $L$ be a Lie algebra over $k,\left(^{3}\right) A$ an abelian subalgebra, and $S(A)$ the symmetric algebra over $A$. We note that $L$ and $L^{*}$ are both modules for $S(A)$. In fact $A$ acts on both $L$ and $L^{*}$ by the adjoint representation; therefore, so does the universal enveloping algebra of $A$, which happens to be $S(A)$ since $A$ is abelian.

We begin with the basic theorem:

Theorem 1. Let $L$ be a Lie algebra over $k, A$ a finite-dimensional abelian subalgebra, and $\alpha$ an element of $L$. Assume that $L^{*}$ is free as a module over $S(A)$. Assume also that $\alpha$ acts semisimply on $L$ and that $A$ is contained in a nonzero eigenspace. Then $H^{i}(L, k)=0$ for $0<i \leq \operatorname{dim} A$.

Proof. Let $m=\operatorname{dim} A$, and consider the Koszul complex:

$$
\begin{aligned}
0 & \rightarrow S(A) \rightarrow S(A) \otimes A^{*} \rightarrow \ldots \rightarrow S(A) \underset{k}{\otimes} \Lambda^{m}\left(A^{*}\right) \\
& \rightarrow S(A) / S^{+}(A) \underset{k}{\otimes} \Lambda^{m}\left(A^{*}\right) \rightarrow 0
\end{aligned}
$$

where $S^{+}(A)$ is the maximal ideal of $S(A)$ of elements of degree $>0$. Taking the tensor product over $S(A)$ with $\Lambda^{i} L^{*}$, which is free over $S(A)$, we get an exact sequence:

$$
\begin{aligned}
0 & \rightarrow \Lambda^{i}\left(L^{*}\right) \otimes A^{*} \stackrel{d}{\longrightarrow} \ldots \\
& \stackrel{d}{\longrightarrow} \Lambda^{i} L^{*} \otimes \Lambda^{m} A^{*} \rightarrow \Lambda^{i} L^{*} / S^{+} \Lambda^{i} L^{*} \otimes \Lambda^{m} A^{*} \rightarrow 0 .
\end{aligned}
$$

Let $\delta$ be the Hochschild coboundary operator for $\Lambda L^{*}$. We can amalgamate the complexes $(2.2)_{i}$ into a double complex, whose $j$ th column is

$$
0 \rightarrow \Lambda^{0} L^{*} \otimes \Lambda^{j} A^{*} \stackrel{\delta}{\longrightarrow} \ldots \rightarrow \Lambda^{k} L^{*} \otimes \Lambda^{j} A^{*} \rightarrow .
$$

We assume $\alpha$ is normalized so that it is $(-1) i d$ on $A$. Then the operator $d$ in (2.2) $)_{i}$ maps $\left(\Lambda^{i} L^{*}\right)_{a} \otimes \Lambda^{j} A^{*}$ into $\left(\Lambda^{i} L^{*}\right)_{a+1} \otimes \Lambda^{j+1} A^{*}$, where the subscript indicates the eigenspace of $\Lambda^{i} L^{*}$ with eigenvalue $a$. Therefore, the double complex we

(2) In [b] Rozenfel'd has announced complete results for (III) $)_{n}$ and $(\mathrm{VI})_{2 n}$. However it is not clear as he claims that the methods of proof in [2] extend in $(\mathrm{VI})_{2 n}$. In [a] Gel'fand et al. show that $(\mathrm{V})_{2}$ has nontrivial cohomology in dimensions 7 and 10.

(3) If $\operatorname{dim} L=\infty$ we assume $L$ is topologized and $L^{* *}=L$. See, for example, [3]. 
have just described breaks up into a countable number of subcomplexes. The $a$ th such complex is described in Figure 1.

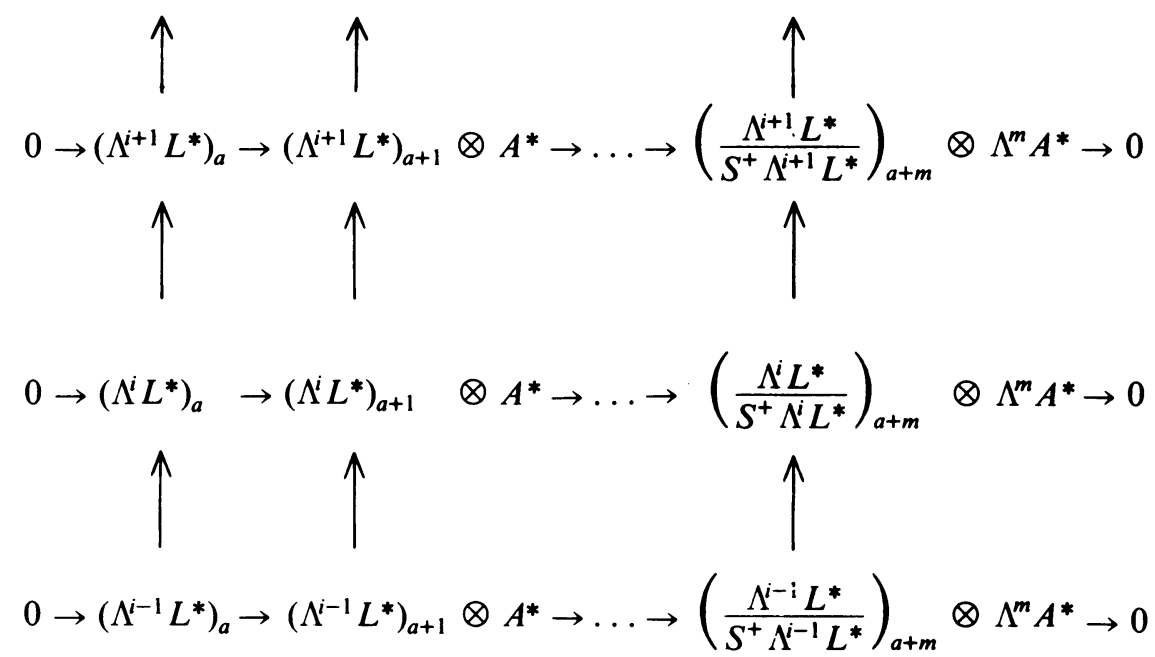

Figure 1

As we have already observed, the rows of Figure 1 are exact. We claim that the columns are exact except for the extreme right-hand column and the column indexed by $a+j=0$. In fact, the standard identity: $(a d \alpha) \omega=\delta(\alpha\lrcorner \omega)+\alpha\lrcorner d \omega$ implies that the only eigenspace of $\Lambda L^{*}$ with nontrivial cohomology is the zero eigenspace.

Let us consider Figure 1 with $a=0$. All the rows are exact, and all the columns are exact except for the extreme right-hand. column. Therefore, we get an isomorphism between the cohomology of the first column in position $i$ (which is just $\left.H^{i}(L, k)\right)$ and the cohomology of the last column in position $i-m$. If $i \leq m$ this cohomology is zero. (It is zero in dimension zero since the complex in question is a relative complex.) Thus $H^{i}(L, k)=0$ for $i \leq m$, proving our theorem.

As corollaries we get:

Corollary 1. The cohomology of the algebra $(\mathrm{I})_{n}$ is zero in dimensions $0<i \leq n$.

Proof. Just take $A$ to be the subalgebra consisting of the constant vector fields, $\sum c_{i}\left(\partial / \partial x_{i}\right), c_{i} \in k$, and take $\alpha=\sum_{i=1}^{n} x_{i}\left(\partial / \partial x_{i}\right)$.

Corollary 2. The cohomology of the algebra $(\mathrm{IV})_{2 n}$ is zero in dimensions $0<i \leq n$. 
Proof. Take $A$ to be the subalgebra consisting of all linear forms $\sum_{i=1}^{n} c_{i} x_{i}$, $c_{i} \in k$, and take $\alpha=\sum x_{i} y_{i}$.

Corollary 3. The cohomology of the algebra (VII) $)_{2 n+1}$ is zero in dimensions $0<i \leq n$.

Proof. Take $A$ and $\alpha$ to be the same as in the preceding example.

3. To apply Theorem 1 to example (II) $n$ we will show that the dual algebra is free over $S(A)$ where $A$ is the subalgebra spanned by $\partial / \partial x_{i}, i=1, \ldots, n-1$. It is not hard to prove this directly, but we prefer to deduce it from a result which is applicable to other examples besides those discussed here.

We begin with a standard theorem in commutative algebra.

Theorem. Let $M$ be a graded module over the polynomial ring $k\left[x_{1}, \ldots, x_{n}\right]$. Then the following are equivalent.

(a) The Koszul cohomology of $M$ with respect to $x_{1}, \ldots, x_{n}$ is zero except in dimension zero.

(b) $M$ is free as a module over $k\left[x_{1}, \ldots, x_{n}\right]$.

(c) For each $j, 0 \leq j \leq n$, $x_{j}$ is a nonzero divisor of $M /\left(x_{1}, \ldots, x_{j-1}\right) M$.

See [1, Chapter VIII, Theorem 6.1] and [6, Chapter IV, Proposition 3].

Using the equivalence of (a) and (b) one can prove some general results relating the vanishing of the cohomology of a Lie algebra, $L$, to the vanishing of its Koszul cohomology with respect to an abelian subalgebra $A$. The Koszul cohomology is in turn closely related to the Spencer cohomology of $L$. (See, for example, [4].)

To show that the cohomology of example (II) $)_{n}$ vanishes in dimensions $0<i<n$ we will use the equivalence of (b) and (c).

Condition (c) dualizes to the following condition:

For each $j$, let $L_{j}=\left\{\vec{f}\right.$, div $\left.\vec{f}=\partial \vec{f} / \partial x_{1}=\cdot=\partial \vec{f} / \partial x_{j-1}=0\right\}$; then the map $L_{j} \rightarrow L_{j}, \vec{f} \rightarrow \partial \vec{f} / \partial x_{j}$, is surjective. To check this condition we note first of all that $L_{j}$ is just all divergence free vector fields in the variables $x_{j}, \ldots, x_{n}$; so it is enough to check the condition for $j=1$. Given $\vec{f} \in L_{1}$ we can find a $\vec{g}$ such that $\left(\partial / \partial x_{1}\right) \vec{g}=\vec{f}$. Since $\operatorname{div} \vec{f}=0,\left(\partial / \partial x_{1}\right) \operatorname{div} \vec{g}=0$; so $\operatorname{div} \vec{g}$ is a power series in $x_{2}, \ldots, x_{n}$. Let $\vec{g}_{1}$ be a power series vector field in $x_{2}, \ldots, x_{n}$ such that $\operatorname{div} \vec{g}_{1}=\operatorname{div} \vec{g}$, then $\left(\partial / \partial x_{1}\right)\left(\vec{g}-\vec{g}_{1}\right)=\vec{f}$ and $\operatorname{div}\left(\vec{g}-\vec{g}_{1}\right)=0$. We have, therefore, proved

Proposition 1. If $L$ is the Lie algebra (II) $)_{n}$ then $L^{*}$ is free over $S(A)$ where $A$ is the subalgebra spanned by $\partial / \partial x_{i}, i=1, \ldots, n-1$.

If $\alpha=\left(\sum_{i=1}^{n-1} x_{i}\left(\partial / \partial x_{i}\right)\right)-(n-1) x_{n}\left(\partial / \partial x_{n}\right)$, the hypotheses of Theorem 1 are satisfied so we have:

Corollary. The cohomology of the algebra (II) $)_{n}$ is zero in dimensions $0<i<n$. 
Remark. One can show that the $n$th cohomology group of (II) $)_{n}$ is onedimensional with the volume form, $d x_{1} \wedge \ldots \wedge d x_{n}$, as its generator. See [7].

4. We will finally compute the cohomology of the algebras (III),$(\mathrm{V})_{2 n}$ and $(\mathrm{VI})_{2 n}$. We begin by describing a spectral sequence due to Hochschild and Serre: Let $L$ be a Lie algebra over $k$ and $M$ an ideal in $L$. The Hochschild-Serre spectral sequence has as its $E_{\infty}$ term $H(L, h)$ and has $E_{2}^{j, i}$ equal to $H^{j}\left(L / M, H^{i}(M)\right)$. (See for example [1, Chapter XVI, §6]).

If $L$ is the Poisson algebra and $M$ is its one-dimensional center, then $H^{i}(M)=k$ when $i=0,1$ and zero otherwise; so $E_{2}^{j, 0}=E_{2}^{j, 1}=H^{j}(L / M, k)$, and the other terms are zero. Since $H^{j}(L, k)=0$ in dimensions $0<j \leq n$ the only way these $E_{2}$ terms can cancel out is for $d_{2}: E_{2}^{j, 1} \rightarrow E_{2}^{j+2,0}$ to be bijective for $j+2 \leq n$. So we have proved

Proposition 2. The cohomology of the Hamiltonian algebra $(\mathrm{V})_{2 n}$ is equal to $k$ in all even dimensions in the range $0 \leq i \leq n$ and equal to zero in all odd dimensions in this range.

Remark. The Poisson algebra is a nontrivial central extension of the Hamiltonian algebra, so it defines an element in $H^{2}$ of the Hamiltonian algebra. (See, for example, [5].) It is not hard to see that the $i$ th power of this 2-dimensional element is a basis for the cohomology in dimension $2 i$ when $2 i \leq n$.

Next we apply the Hochschild-Serre spectral sequence to the Hamiltonian algebra and to its derivation algebra. Let $M$ be the Hamiltonian algebra and $L$ its derivation algebra. It is easy to see that the two-dimensional generator of $H(M)$ is not invariant with respect to $L / M$, so $H^{i}\left(L / M, H^{j}(M)\right)$ is zero except when $j=0$; and $H^{i}\left(L / M, H^{0}\right)$ is equal to $k$ for $i=0,1$ and zero otherwise, since $L / M$ is one-dimensional. We conclude with

Proposition 3. The cohomology of the algebra $(\mathrm{VI})_{2 n}$ is equal to $k$ in dimensions zero and one and is zero in the range $1<i \leq n$.

A simpler computation of the same kind shows

Proposition 4. The cohomology of the algebra (III) $)_{n}$ is equal to $k$ in dimensions zero and one, and is zero in the range $1<i \leq n$.

Remark. The generator of the first cohomology group of (III) $)_{n}$ is the one cocycle $\vec{f} \rightarrow \operatorname{div} \vec{f}$. The restriction of this is the generator of the first cohomology group of $(\mathrm{VI})_{2 n}$.

To conclude, we note that Gel'fand and Fuks have proved the cohomology of (I) $n$ vanishes in the range $0<i \leq 2 n$. See [2]. The second author, in his thesis, has proved that the cohomology of (IV $)_{2 n}$ also vanishes in this range. (The proofs require rather complicated techniques from classical invariant theory.) 


\section{BIBLIOGRAPHY}

1. H. Cartan and S. Eilenberg, Homological algebra, Princeton Univ. Press, Princeton, N. J., 1956. MR 17, 1040.

2. I. M. Gel'fand and D. B. Fuks, Cohomology of the Lie algebra of formal vector fields, Izv. Akad. Nauk SSSR Ser. Mat. 34 (1970), 322-337 = Math. USSR Izv. 4 (1970), 327-341. MR 42 \# 1103.

3. V. Guillemin, A Jordan-Hölder decomposition for a certain class of infinite dimensional Lie algebras,

J. Differential Geometry 2 (1968), 313-345. MR 41 \#8481.

4. V. Guillemin and S. Sternberg, An algebraic model of transitive differential geometry, Bull. Amer. Math. Soc. 70 (1964), 16-47. MR 30 \#333.

5. N. Jacobson, Lie algebras, Interscience Tracts in Pure and Appl. Math., no. 10, Interscience, New York, 1962. MR 26 \# 1345.

6. J.-P. Serre, Algèbre locale. Multiplicités, Cours au Collège de France, 1957/58, 2ième éd., Lecture Notes in Math., vol. 11, Springer-Verlag, Berlin and New York, 1965. MR 34 \# 1352.

7. S. Shnider, Invariant theory and the cohomology of infinite dimensional Lie algebras, Thesis, Harvard University, Cambridge, Mass., 1972.

ADDED IN PROOF.

a. I. M. Gel'fand, D. I. Kalinin and D. B. Fuks, On the cohomology of the Lie algebra of Hamiltonian formal vector fields, Funkcional. Anal. i Priložen. 6 (1972), 25-29. (Russian)

b. B. I. Rozenfel'd, Cohomologies of some infinite dimensional Lie algebras, Funkcional. Anal. i Priložen. 5 (1971), 84-85. (Russian)

Department of Mathematics, Massachusetts Institute of Technology, Cambridge, MASSACHUSETTS 02139

Department of Mathematics, Harvard University, Cambridge, Massachusetts 02138 